\title{
Assigning the NH stretches of the guanine tautomers using adiabatic separation: $\operatorname{CCSD}(\mathrm{T})$ benchmark calculations
}

\author{
Dana Nachtigallová, Pavel Hobza and Vladimír Špirko \\ Center for Biomolecules and Complex Molecular Systems \\ Institute of Organic Chemistry and Biochemistry, v.v.i., \\ Academy of Sciences of the Czech Republic \\ Flemingovo nám. 2, 16010 Prague 6, Czech Republic
}


TABLE I: Relative energies (in $\mathrm{kJ} / \mathrm{mol}$ ) of the guanine tautomers

\begin{tabular}{lcccc}
\hline & MP2/cc-pVTZ & CCSD $(\mathrm{T})^{a}$ & B3LYP/cc-pVTZ CCSD(T)/aug-cc-pVDZ \\
\hline G7K & 0.00 & 0.00 & 0.00 & 0.00 \\
G9K & 1.57 & 1.80 & 2.16 & 2.89 \\
G9Eb & 1.86 & 1.76 & 7.01 & 3.81 \\
G9Ea & 5.05 & 5.54 & 9.38 & 5.48 \\
G7E & 14.90 & 14.71 & 19.55 & 15.48 \\
G7Im & 30.30 & 27.65 & 24.24 & \\
\hline
\end{tabular}

${ }^{a}$ Optimized at the MP2/cc-pVTZ level ${ }^{b}$ Optimized at the MP2/aug-cc-pVDZ level ${ }^{1}$

TABLE II: Observed and calculated NH vibrational frequencies ${ }^{a}$

\begin{tabular}{|c|c|c|c|c|c|c|c|c|c|}
\hline \multicolumn{3}{|c|}{ Experiment } & $\mathrm{MP} 2^{b}$ & \multicolumn{2}{|l|}{$\mathrm{MP} 2^{c}$} & \multicolumn{2}{|c|}{$\operatorname{CCSD}(\mathrm{T})^{c} \operatorname{BaLYP}^{c}$} & \multirow[b]{2}{*}{$\mathrm{PT}^{f}$} & \multirow[b]{2}{*}{$\mathrm{AS}^{e}$} \\
\hline & Ref. $^{2}$ & Ref. ${ }^{3}$ Ref. $^{4}$ & $\mathrm{HA}^{d}$ & $\mathrm{HA}^{d}$ & $\mathrm{AS}^{e}$ & $\mathrm{AS}^{e}$ & $\mathrm{HA}^{d}$ & & \\
\hline G7K & $3504.8(120)$ & 35043497 & 3660.6 & 3668.0 & 3525.4 & $3529.6(102)$ & $3650.3(92)$ & 3470.5 & 3505.9 \\
\hline G7Im & & 3505 & & 3666.6 & 3524.8 & $3529.2(100)$ & $3649.6(106)$ & 3475.9 & 3506.4 \\
\hline G9K & $3506.9(100)$ & 3490 & 3664.8 & 3669.7 & 3529. & $3530.4(85)$ & $3647.1(69)$ & 3484.0 & 3504.2 \\
\hline G9Eb & $3509.6(105)$ & 3508 & 3667.4 & 3673.6 & 3533.1 & $3534.1(83)$ & $3651.7(72)$ & 3495.2 & 3509.1 \\
\hline G9Ea & $3511.3(104)$ & 35083516 & 3669.6 & 3675.7 & 3535.0 & $3535.3(84)$ & $3652.5(73)$ & 3488.8 & 3510.1 \\
\hline G7E & & 3516 & & 3682.0 & 3540.1 & $3542.0(100)$ & $3662.3(84)$ & 3481.2 & 3518.2 \\
\hline
\end{tabular}

${ }^{a}$ Values given in parentheses are absolute IR intensities (in $\mathrm{km} / \mathrm{mol}$ ); the IR intensities taken from Ref. ${ }^{2}$ were calculated using harmonic approximation. ${ }^{b} 6-311 \mathrm{G}^{*}$ basis set ${ }^{2},{ }^{c} \mathrm{cc}-\mathrm{pVTZ}$ basis set, ${ }^{d}$ harmonic approximation, ${ }^{e}$ adiabatic separation, ${ }^{f}$ perturbation theory.

1 Hanuš, M.; Ryjáček, F.; Kabeláč, M.; Kubař, T.; Bogdan, T. V.; Trygubenko, S. A.; Hobza, P. J. Am. Chem. Soc. 2003, 125, 7678-7688.

2 Choi, M. Y.; Miller, R. E. J. Am. Chem. Soc. 2006, 128, 7320-7328.

3 Mons, M.; Dimicoli, I.; Piuzzi, F.; Tardivel, B.; Elhanine, M. J. Phys. Chem. A 2002, 106, 5088-5094.

4 Nir, E.; Plutzer, C.; Kleinermanns, K.; de Vries, M. S. Eur. Phys. J. D 2002, 20, 317-329. 
TABLE III: Observed and calculated $\mathrm{NH}_{2}$ vibrational frequencies of $\mathrm{G} 7 \mathrm{~K}$ and $\mathrm{G}^{2} \mathrm{~K}^{a}$

\begin{tabular}{|c|c|c|c|c|c|c|}
\hline & Experiment & $\mathrm{MP}^{b}$ & $\mathrm{MP} 2^{c}$ & $\operatorname{CCSD}(\mathrm{T})^{c}$ & B3LYH & \\
\hline & $\operatorname{Ref}^{2}$ & $\mathrm{HA}^{d}$ & $\mathrm{AS}^{e}$ & $\mathrm{AS}^{e}$ & $\mathrm{HA}^{d}$ & $\mathrm{PT}^{f}$ \\
\hline G9K: ${ }^{a s} \nu_{N H_{2}}$ & 3544.5 & 3701.3 & 3500.8 & 3466.1 & 3680.6 & 3522.2 \\
\hline G9K: ${ }^{s y m} \nu_{N H_{2}}$ & 3444.5 & 3587.7 & 3401.5 & 3370.4 & 3572.4 & 3428.5 \\
\hline $\mathrm{G} 7 \mathrm{~K}:{ }^{a s} \nu_{N H_{2}}$ & 3526.6 & 3687.1 & 3486.8 & 3452.5 & 3667.3 & 3505.6 \\
\hline $\mathrm{G} 7 \mathrm{~K}:{ }^{s y m} \nu_{N H_{2}}$ & 3430.5 & 3576.2 & 3391.8 & 3358.5 & 3561.8 & 3415.9 \\
\hline
\end{tabular}

${ }^{a}$ The dynamical calculations were simplified by using the leading (constant) terms of the standard G matrix. ${ }^{b} 6-311 \mathrm{G}^{*}$ basis set, ${ }^{2}{ }^{c} \mathrm{cc}-\mathrm{pVTZ}$ basis set, ${ }^{d}$ harmonic approximation, ${ }^{e}$ adiabatic separation, ${ }^{f}$ perturbation theory. 